\title{
Predictors of clinically relevant bleeding and overdose in prospectively followed cohort of dabigatran treated stroke patients
}

Tomek Ales ${ }^{1,2}$, Olserova Anna ${ }^{1}$, Boudnikova Anna ${ }^{1}$, Jansky Petr ${ }^{1}$, Kesnerova Petra ${ }^{1}$, Kaplan Vojtech ${ }^{2}$, Lacinova Zuzana ${ }^{2}$, Ruzicková Tereza ${ }^{1}$, Magerova Hana ${ }^{1}$, Sarbochová Ivana ${ }^{1}$, Klima Jan³, Matoska Vaclav

1) Department of Neurology, 2nd Medical Faculty of Charles University and Motol University Hospital, Prague, Czech Republic . 2) Molecular Genetics Laboratory, Hospital Na Homolce, Prague, Czech Republic. 3) Pharmakl, Prague, Czech Republic

\section{Background}

Dabigatran is direct thrombin inhibitor approved in the prevention of stroke in patients with AF. It was shown in the RELY trial substudy (1) that genetic variants could contribute to interindividual variability in concentrations of the active metabolite of dabigatran and influence the safety of treatment.

Carriage of the CES1 rs2244613 minor allele was in RE-LY pharmacogenetics substudy associated with lower exposure to active metabolite and with a lower bleeding risk compared to wild-type allele (1).

\section{Aim of the study}

To determine the influence of CES1 polymorphism rs2244613 on occurrence of clinically relevant bleeding (CRB) or higher plasmatic levels in dabigatran treated stroke patients.

\section{Methods}

Prospective observational monocentric (Comprehensive stroke centre) study in consenting cardioembolic stroke patients initiated on dabigatran.

Primary outcome was clinically relevant bleeding (CRB) defined as composite of major bleeding (ISTH criteria), clinically relevant non-major bleeding and/or dabigatran through plasmatic level $c_{\min }>300 \mathrm{ng} / \mathrm{ml}$.

Secondary outcomes were death from any cause, recurrent ischemic stroke/TIA/myocardial infarction.

\section{Bleeding definitions}

ISTH major bleeding in non-surgical patients:

1) Fatal bleeding. 2) Bleeding in a critical area or organ, 3) Bleeding causing a fall in haemoglobin level of $20 / \mathrm{L}$ or leading to transfusion of 2 or more units Clinically relevant non-major bleeding: Bleeding episode not meeting ISTH definition of major bleeding but does meet at least 1 of the following criteria: 1) requiring medical intervention by a healthcare professional. 2) leading to hospitalization or increased level of care. 3) prompting a face-to-face healthcare professional evaluation.

DNA analysis of CES1 polymorphism rs2244613 was done with RFLP analysis.

Through concentration of dabigatran $\left(c_{\min }\right)$ was measured with liquid chromatography-tandem mass spectrometry (LC-MS/MS) at least 7 days after initiation, therapeutic range $50-200 \mathrm{ng} / \mathrm{ml}$. Overdose was defined as $\mathrm{c}_{\mathrm{min}}>300 \mathrm{ng} / \mathrm{ml}$.

Statistical analysis - T-test, Chi2, Fisher exact, Mann Whitney U test, Cox regression, Kaplan Meier (SPSS 24, IBM).

\section{Results}

110 patients after cardioembolic stroke were enrolled and followed up for mean 19.9 months.

\begin{tabular}{|c|c|c|c|}
\hline & $\begin{array}{l}\text { Clinically relevant } \\
\text { bleeding and overdose } \\
\text { group }(n=12)\end{array}$ & $\begin{array}{l}\text { Group without clinically } \\
\text { relevant bleeding and } \\
\text { overdose }(n=98)\end{array}$ & $\begin{array}{l}\text { Difference } \\
\text { between } \\
\text { groups }\end{array}$ \\
\hline Age, years; mean (SD) & 75.1 (7.9) & $69.6(13.0)$ & 0.109 \\
\hline Male sex & $6(50 \%)$ & $50(51 \%)$ & 1.0 \\
\hline Weight, kg; mean (SD) & $79.9(16.0)$ & $80.4(1.5)$ & \\
\hline $\begin{array}{l}\text { Follow-up time, months; } \\
\text { mean (SD) }\end{array}$ & $8.6(10.3)$ & $21.3(19.6)$ & $0.007^{*}$ \\
\hline $\mathrm{CHA}_{2} \mathrm{DS}_{2}-\mathrm{VASc}$; mean (SD) & $5.8(1.1)$ & 4.8 (1.9) & $0.032 *$ \\
\hline HAS-BLED; mean (SD) & $3.7(1.5)$ & $3.2(1.6)$ & 0.819 \\
\hline Hypertension & $11(91.7 \%)$ & $71(72.4 \%)$ & 0.290 \\
\hline Diabetes mellitus & $4(33.3 \%)$ & $32(32.7 \%)$ & 1.0 \\
\hline $\begin{array}{l}\text { Heart failure, ejection } \\
\text { fraction of left ventricle } \\
<35 \%\end{array}$ & $3(25.0 \%)$ & $13(13.3 \%)$ & 0.208 \\
\hline $\begin{array}{l}\text { Serum creatinine, umol/Li } \\
\text { mean (SD) }\end{array}$ & $88.4(20.6)$ & $80.2(22.5)$ & 0.898 \\
\hline CES 1 variant allele & $1(8.3 \%)$ & $41(41.8 \%)$ & $0.028 *$ \\
\hline Ischemic stroke/TIA/MI & $2(16.7 \%)$ & $10(10.2 \%)$ & 0.618 \\
\hline Mortality, any cause & $2(16.7 \%)$ & $4(4.1 \%)$ & 0.445 \\
\hline Dosage $150 \mathrm{mg}$ bid & $6(50.0 \%)$ & $72(73.5 \%)$ & 0.104 \\
\hline
\end{tabular}

There were 8 episodes of $C R B$ and 4 patients with $c_{\min }$ above $300 \mathrm{ng} / \mathrm{ml}$. There were 8 episodes of clinically relevant bleeding, all in patients with wild-type genotype: $3 \times$ gastrointestinal, retroperitoneal, intracerebral, $2 \times$ subcutaneous and hemoptysis. 5 patients died: intracerebral bleeding, cardiac arrest, $2 \mathrm{X}$ sepsis + pneumonia, 1 x unknown cause.

Carriers of minor allele were less likely to have the outcome: HR $0.124(95 \% \mathrm{Cl} 0.02-0.96, \mathrm{p}=0.046)$.

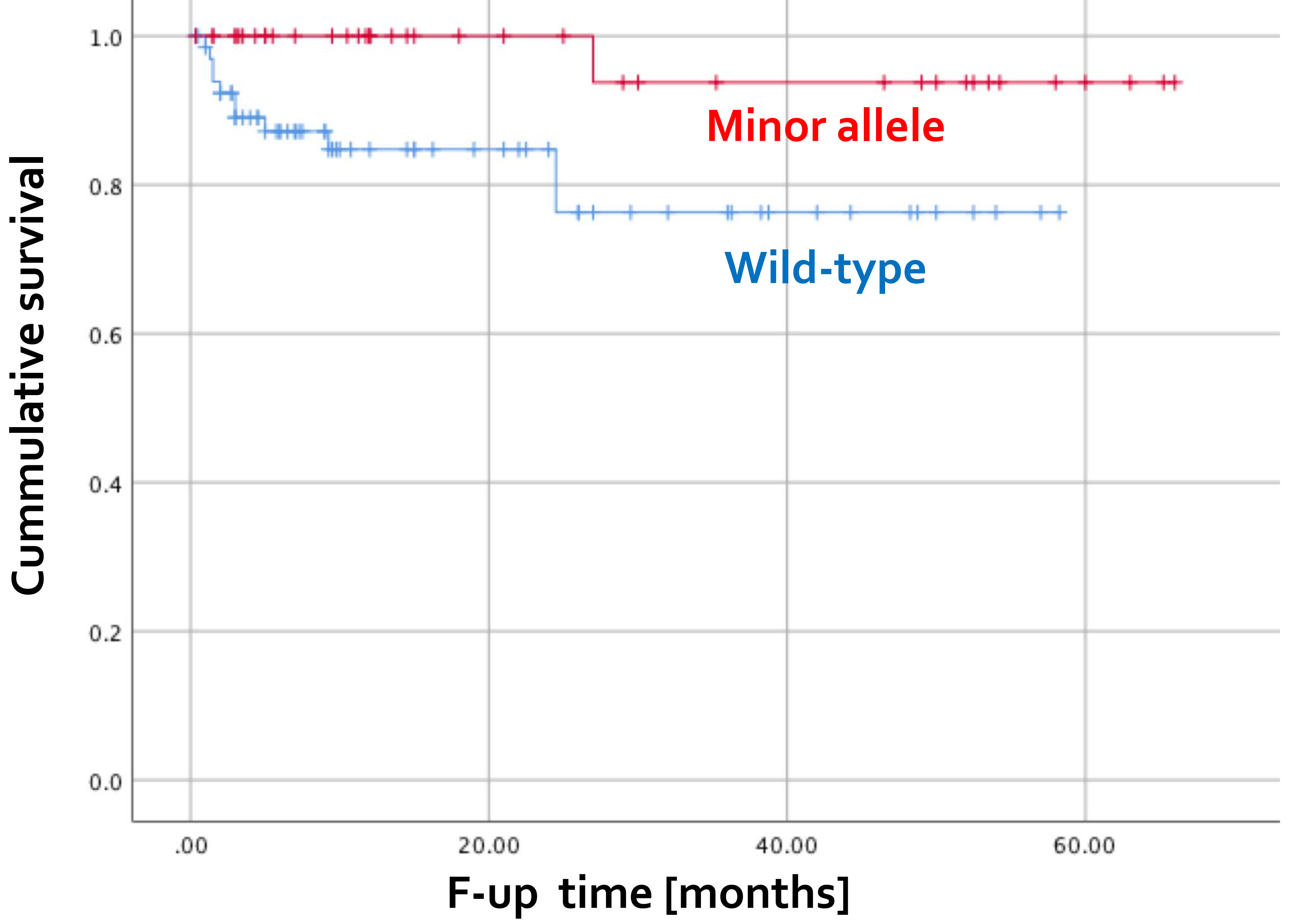

